Measurement and Reproducibility of Preserved Ellipsoid Zone Area and Preserved Retinal Pigment Epithelium Area in Eyes With Choroideremia

\author{
Hariri, Amir H.
}

2017-07

Hariri , A H , Velaga , S B , Girach , A , Ip , M S , Le , P V , Lam , B L , Fischer , M D , Sankila , E-M , Pennesi , M E , Holz, F G, Maclaren , R E, Birch , D G, Hoyng , C B , Macdonald, I M , Black, G C , Tsang , S H , Bressler, N M , Larsen , M , Gorin , M B , Webster , A R , Sadda , S R \& Nat Hist Progression Choroideremia 2017 , ' Measurement and Reproducibility of Preserved Ellipsoid Zone Area and Preserved Retinal Pigment Epithelium Area in Eyes With Choroideremia ' , American Journal of Ophthalmology , vol. 179 , pp. 110-117 . https://doi.org/10.1016/j.ajo.2017.05.002

http://hdl.handle.net/10138/297802

https://doi.org/10.1016/j.ajo.2017.05.002

publishedVersion

Downloaded from Helda, University of Helsinki institutional repository.

This is an electronic reprint of the original article.

This reprint may differ from the original in pagination and typographic detail.

Please cite the original version. 


\title{
Measurement and Reproducibility of Preserved Ellipsoid Zone Area and Preserved Retinal Pigment Epithelium Area in Eyes With Choroideremia
}

\author{
AMIR H. HARIRI, SWETHA B. VELAGA, ANIZ GIRACH, MICHAEL S. IP, PHUC V. LE, BYRON L. LAM, \\ M. DOMINIK FISCHER, EEVA-MARJA SANKILA, MARK E. PENNESI, FRANK G. HOLZ, ROBERT E. MACLAREN, \\ DAVID G. BIRCH, CAREL B. HOYNG, IAN M. MACDONALD, GRAEME C. BLACK, STEPHEN H. TSANG, \\ NEIL M. BRESSLER, MICHAEL LARSEN, MICHAEL B. GORIN, ANDREW R. WEBSTER, AND SRINIVAS R. SADDA, \\ FOR THE NATURAL HISTORY OF THE PROGRESSION OF CHOROIDEREMIA (NIGHT) STUDY GROUP
}

- PURPOSE: To identify valid and reproducible methods for quantifying anatomic outcome measures for eyes with choroideremia (CHM) in clinical trials.

- DESIGN: Reliability analysis study.

- METHODS: In this multicenter study, patients with confirmed genetic diagnosis of $\mathrm{CHM}$ were enrolled. All cases underwent spectral-domain optical coherence tomography (SDOCT) and fundus autofluorescence (FAF) imaging. Two graders independently delineated boundaries of preserved autofluorescence (PAF) and pre-

\footnotetext{
AJO.com

Accepted for publication May 1, 2017

From the Doheny Image Reading Center, Doheny Eye Institute, Los Angeles, California (A.H.H., S.B.V., M.S.I., P.V.L., S.R.S.); Department of Ophthalmology, David Geffen School of Medicine of the University of California-Los Angeles, Los Angeles, California (A.H.H., M.S.I., P.V.L., M.B.G., S.R.S.); NightstaRx Ltd, London, United Kingdom (A.G.); Department of Ophthalmology, Bascom Palmer Eye Institute, University of Miami Miller School of Medicine, Miami, Florida (B.L.L.); Centre for Ophthalmology, University of Tübingen, Tübingen, Germany (M.D.F.); Helsinki University Eye Hospital, Finland (E.-M.S.); Casey Eye Institute, Oregon Health \& Science University, Portland, Oregon (M.E.P.); Department of Ophthalmology, University of Bonn, Bonn, Germany (F.G.H.); Nuffield Laboratory of Ophthalmology, Department of Clinical Neurosciences, University of Oxford and Oxford University Eye Hospital, NHS Foundation Trust, NIHR Biomedical Research Centre, Oxford, United Kingdom (R.E.M.); Moorfields Eye Hospital, NHS Foundation Trust, NIHR Biomedical Research Centre, London, United Kingdom (R.E.M.); Retina Foundation of the Southwest, Dallas, Texas (D.G.B.); Department of Ophthalmology, Radboud University Medical Center, Nijmegen, Netherlands (C.B.H.); Department of Ophthalmology and Visual Sciences, University of Alberta, Edmonton, Alberta, Canada (I.M.M.); Manchester Centre for Genomic Medicine, Central Manchester University Hospitals NHS Foundation Trust, Manchester Academic Health Sciences Centre, St Mary's Hospital, Manchester, United Kingdom (G.C.B.); Departments of Ophthalmology and of Pathology and Cell Biology, Columbia University, New York, New York (S.H.T.); Wilmer Eye Institute, Johns Hopkins University School of Medicine, Baltimore, Maryland (N.M.B.); Department of Ophthalmology, Rigshospitalet and University of Copenhagen, Copenhagen, Denmark (M.L.); Moorfields Eye Hospital NHS Foundation Trust, London, United Kingdom (A.R.W.); and UCL Institute of Ophthalmology, London, United Kingdom (A.R.W.).

Inquiries to SriniVas R. Sadda, President and Chief Scientific Officer, Doheny Eye Institute, David Geffen School of Medicine, University of California, Los Angeles, California; e-mail: ssadda@doheny.org
}

served ellipsoid zone (EZ) on FAF and OCT images, respectively. The results of the 2 independent gradings of both FAF and OCT images were compared to assess the reproducibility of the grading methods.

- RESULTS: A total of 148 eyes from 75 cases were included. In $21 \%$ of eyes PAF and in $43 \%$ of eyes preserved EZ had extended beyond the image capture area. After exclusion of these eyes and low-quality images, 114 FAF and 77 OCT images were graded. The mean PAF areas from 2 independent gradings were $3.720 \pm 3.340 \mathrm{~mm}^{2}$ and $3.692 \pm 3.253 \mathrm{~mm}^{2}$, respectively. Intraclass correlation coefficient (ICC) for these gradings was 0.996 . The mean preserved EZ areas from 2 independent gradings were $2.746 \pm 2.319 \mathrm{~mm}^{2}$ and $2.858 \pm 2.446 \mathrm{~mm}^{2}$, respectively. ICC for these gradings was 0.991 .

- CONCLUSIONS: Quantifying preserved retinal pigment epithelium and EZ areas on FAF and OCT images, respectively, in CHM patients is highly reproducible. These variables would be potential anatomic outcome measures for CHM clinical trials and could be studied and tracked longitudinally in choroideremia. (Am J Ophthalmol 2017;179:110-117. (C) 2017 Elsevier Inc. All rights reserved.)

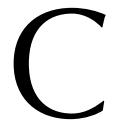
HOROIDEREMIA (CHM) IS AN X-LINKED RECESSIVE, progressive retinal degenerative disease with an estimated prevalence of 1 in 50000 to 1 in 100000 individuals, ${ }^{1}$ with the highest prevalence in Finland. ${ }^{2}$

$\mathrm{CHM}$ is caused by null mutations in the CHM gene (Xq21), which leads to the absence of an intracellular protein called Rab escort protein-1 (REP1)., ${ }^{3,4}$ As a result, progressive but slow degenerative changes in the neurosensory retina, retinal pigment epithelium (RPE), and choroid ${ }^{5}$ start from the periphery and expand centripetally. Patients usually have good visual acuity until degeneration encroaches on the fovea. ${ }^{6}$ Of note, before age 40 , many patients retain 20/20 visual acuity. ${ }^{7}$ There is still uncertainty whether the neuroretina, RPE, and choroid are all primarily affected, or whether one or more of these 
layers is secondarily affected. ${ }^{8}$ It is perhaps not surprising that deficiency of the CHM gene product leads to a functional impairment before RPE cell death, but it is not clear whether this is related specifically to deficiency or if it is a nonspecific consequence of general stress to the RPE cells. ${ }^{9}$

Even though the sequence of events that leads to visual dysfunction is not fully understood, recently gene replacement in CHM patients has been explored clinically, and the first reports were promising., ${ }^{6,10}$ Improvement in visual acuity was observed in a subset of patients ( 2 out of 6) after this gene therapy. However, as mentioned above, visual acuity does not decrease until very late in the disease course. Thus, by relying on visual acuity as the only outcome measure, just very advanced CHM cases would be enrolled in interventional clinical trials. Therefore, other outcome measures, either anatomic or physiological or both, should be applied in clinical trials of CHM. These outcome measures could be the area of preserved RPE, preserved ellipsoid zone (EZ), and preserved choroid. In addition, neurosensory retinal volume and microperimetric retinal sensitivity are other potential outcome measures.

Seitz and associates ${ }^{11}$ reported that the area of preserved RPE on fundus autofluorescence (FAF) images and the neurosensory retinal volume measured on optical coherence tomography (OCT) images demonstrate some symmetry between the eyes of the same subject with CHM. In another very recent report, FAF images from CHM patients were used to evaluate progression of the disease. ${ }^{12}$

In this study, taking advantage of the largest-ever natural history study of the choroideremia, we have identified reproducible methods for grading of FAF and OCT to quantify the area of preserved RPE and preserved EZ, respectively. This study also addresses grading challenges that may be encountered during CHM clinical trials.

\section{METHODS}

IN THIS MULTICENTER STUDY, SUBJECTS WITH A CLINICAL phenotype of $\mathrm{CHM}$ and confirmed genetic diagnosis were enrolled. All the subjects were male, were at least 18 years old, and met visual acuity eligibility criteria of 34-88 ETDRS letters (corresponding to 20/20 to 20/200 Snellen equivalent) in at least one eye. All subjects provided informed consent to participate in the study. Only cases with visible chorioretinal atrophy within the vascular arcades at the posterior pole were included. All eyes with any concomitant retinal pathology were excluded from the study. All participants underwent a complete ocular examination, spectraldomain OCT (SDOCT), color fundus photography, microperimetry, and FAF imaging at the same time. The study was approved by the institutional review board of the University of California, Los Angeles. The research adhered to the tenets set forth in the Declaration of Helsinki.
Both FAF and SDOCT images were captured by the Heidelberg Retinal Angiograph or Spectralis (Heidelberg Engineering, Heidelberg, Germany) systems. Scan angle of FAF images was 30 degrees. SDOCT images were obtained with a macular cube protocol of $1024 \mathrm{~A}$-scans $\times 97 \mathrm{~B}$-scans over a $6 \mathrm{~mm} \times 6 \mathrm{~mm}$ area centered on the foveal center.

Two certified Doheny Image Reading Center (DIRC) graders independently delineated boundaries of preserved autofluorescence (PAF) on all the FAF images, taking advantage of the Heidelberg Spectralis Region Finder tool (Figure 1). In addition, the more senior grader graded the same cases in a different session days later to document the intragrader reproducibility. When the PAF extended beyond the captured area of FAF, the case was excluded. The minimum size of the PAF islands that were included in grading was $0.1 \mathrm{~mm}^{2}$ (>175 $\mu \mathrm{m}$ in longest linear dimension, which is equal to the diameter of a blood vessel). This tool compensated for image scaling differences; thus it is an efficient tool in longitudinal studies to eliminate the scaling noise that otherwise may be encountered. ${ }^{13}$

To grade OCT scans, all volume scans were initially inspected to exclude the eyes with extension of the EZ band to the nasal or temporal border of any B-scan, or if the EZ was visible on the most inferior or superior B-scan. ${ }^{14}$ Then the OCT volume cubes were exported from the instruments and loaded into 3D-OCTOR, version 3 , a custom OCT grading software program developed by the DIRC that has previously been described and validated. ${ }^{15,16}$ To quantify the area of preserved EZ for this analysis, 2 certified DIRC graders independently segmented the EZ band on all B-scans. Also, similar to FAF grading, the more senior grader performed another independent grading session. The software then computed the area confined between the preserved EZ band and Bruch membrane (for every A-scan) and interpolated between adjacent B-scans to generate a preserved EZ area map (Figure 2). We adopted the following guidelines from Jacobson and associates ${ }^{5}$ that helped our graders to spot the edges of the preserved EZ band in challenging cases: (1) interlaminar bridges that mark the abrupt transition from central relatively intact retinal lamination to outer retinal atrophy; (2) a hyperreflective band from the external limiting membrane and the ellipsoid band to the outer nuclear layer plus the Henle fiber layer at the edge of the preserved EZ band; and (3) abrupt change in sub-RPE illumination at the border between preserved and atrophic RPE.

Most of the choroideremia cases had multiple foci of RPE hyperplasia that could be misinterpreted as preserved EZ area. To differentiate these areas from preserved EZ, graders paid particular attention to the choroid appearance exactly underneath the questionable area. Pigment hyperplasia causes a prominent shadowing of choroid, but those parts of the choroid that are beneath preserved EZ are not shadowed and are sometimes even enhanced (Figure 3). 

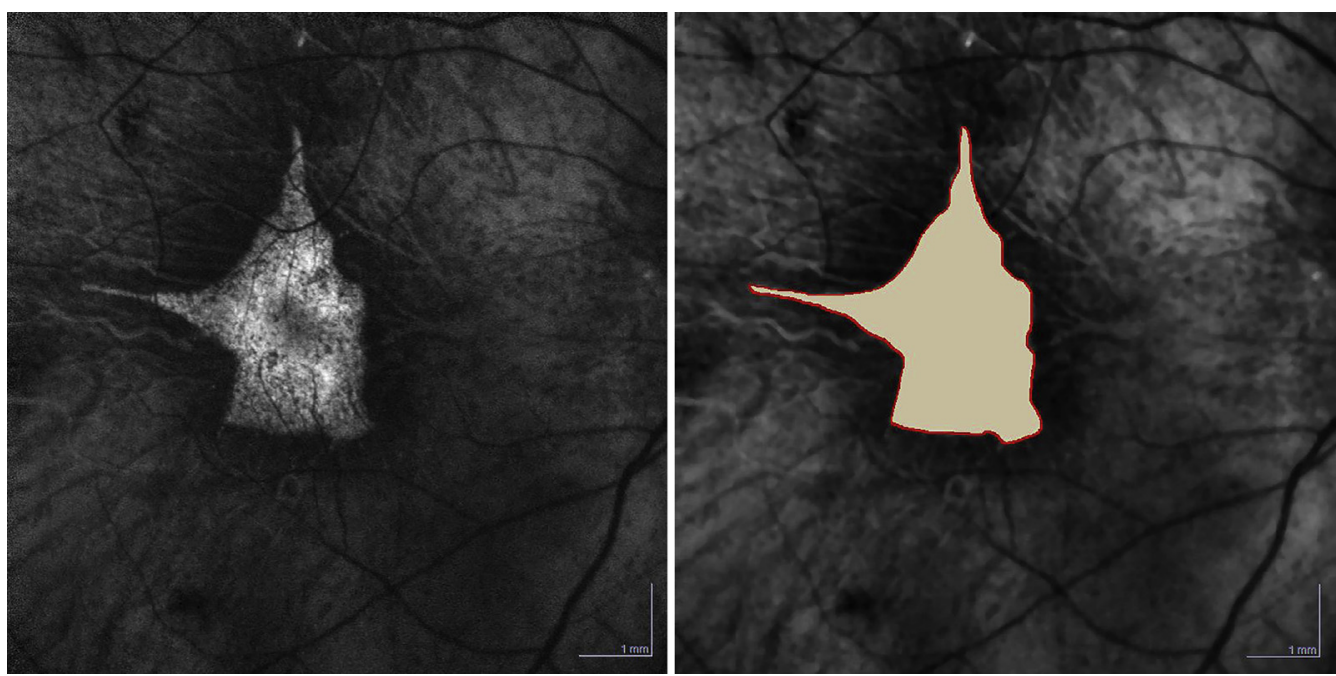

FIGURE 1. (Left) Fundus autofluorescence image of a choroideremia patient. (Right) Boundaries of the preserved autofluorescence have been delineated by taking advantage of the Heidelberg Spectralis Region Finder tool.
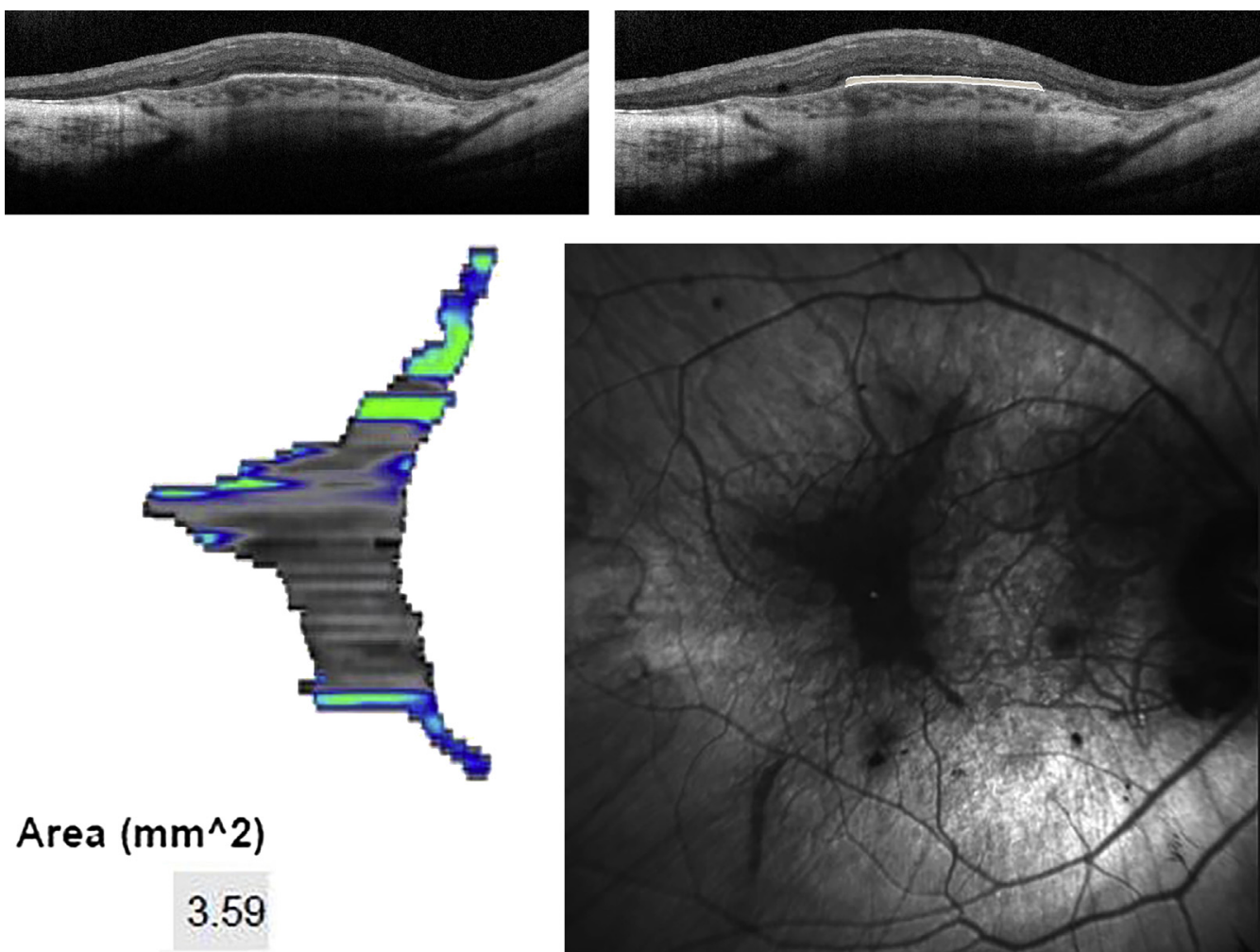

FIGURE 2. (Top, left and right) Segmenting the ellipsoid zone band on a regular B-scan using 3D-OCTOR, a custom optical coherence tomography grading software program. (Bottom left) The software then computes the area confined between the preserved ellipsoid zone band and Bruch membrane (for every A-scan) and interpolates between adjacent B-scans to generate a preserved ellipsoid zone area map. (Bottom right) Corresponding infrared image is shown for comparison.

- STATISTICAL ANALYSIS: We compared the results of the independent grading sessions. Intergrader and intragrader reproducibility were assessed with the intraclass correlation coefficient (ICC). A higher ICC indicates better reproducibility of the variable. We also generated Bland-Altman plots to show the agreement between the 

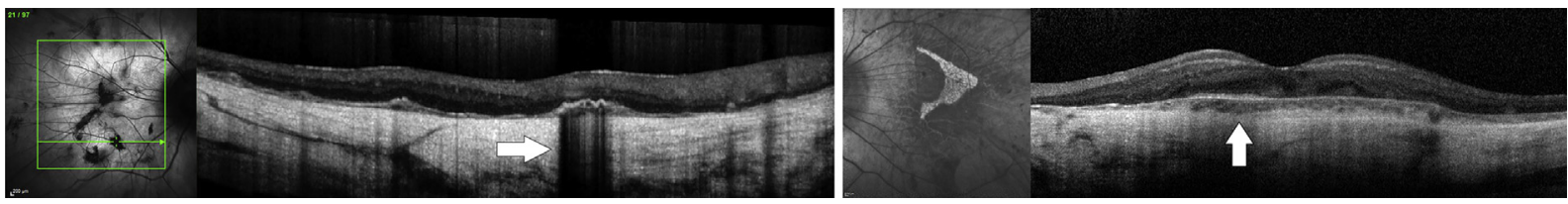

FIGURE 3. To differentiate retinal pigment epithelium hyperplasia from the preserved ellipsoid zone, evaluating underneath choroid is crucial. Pigment hyperplasia causes a prominent shadowing of the choroid (Left, white horizontal arrow), but those parts of the choroid that are beneath the preserved ellipsoid zone can be seen as normal and preserved choroid (Right, white vertical arrow).

TABLE 1. The Preserved Autofluorescence and Preserved Ellipsoid Zone Areas Measured by the 2 Independent Graders and Comparison Between These Independent Grading Sessions

\begin{tabular}{lcc}
\hline & Preserved Autofluorescence Area & Preserved Ellipsoid Zone Area \\
\hline Number of eyes & 114 & 77 \\
Grader 1 (mean \pm SD) & $3.720 \pm 3.340 \mathrm{~mm}^{2}$ & $2.746 \pm 2.319 \mathrm{~mm}^{2}$ \\
Grader 2 (mean \pm SD) & $3.692 \pm 3.253 \mathrm{~mm}^{2}$ & $2.858 \pm 2.446 \mathrm{~mm}^{2}$ \\
Difference (mean \pm SD) & $0.029 \pm 0.279 \mathrm{~mm}^{2}$ & $0.109 \pm 0.317 \mathrm{~mm}^{2}$ \\
Absolute difference (mean \pm SD) & $0.144 \pm 0.241 \mathrm{~mm}^{2}$ & $0.144 \pm 0.303 \mathrm{~mm}^{2}$ \\
Coefficient of variation & 1.67 & 2.1 \\
Percentage difference (mean \pm SD) & $6.61 \pm 10.10$ & $7.84 \pm 13.83$ \\
Intraclass correlation coefficient & $0.996(95 \% \mathrm{Cl}, 0.995-0.998)$ & $0.991(95 \% \mathrm{Cl}, 0.986-0.994)$ \\
\hline
\end{tabular}

TABLE 2. Comparison Between the Preserved Autofluorescence and Preserved Ellipsoid Zone Areas Measured by 1 Senior Grader in 2 Independent Sessions

\begin{tabular}{lcc}
\hline & Preserved Autofluorescence Area & Preserved Ellipsoid Zone Area \\
\hline Number of eyes & 114 & 77 \\
Difference (mean \pm SD) & $0.001 \pm 0.114 \mathrm{~mm}^{2}$ & $0.017 \pm 0.151 \mathrm{~mm}^{2}$ \\
Absolute difference (mean \pm SD) & $0.079 \pm 0.082 \mathrm{~mm}^{2}$ & $0.089 \pm 0.123 \mathrm{~mm}^{2}$ \\
Coefficient of variation & 1.04 & 1.38 \\
Percentage difference (mean \pm SD) & $3.91 \pm 4.19$ & $4.43 \pm 5.28$ \\
Intraclass correlation coefficient & $0.999(95 \% \mathrm{Cl}, 0.999-1.00)$ & $0.998(95 \% \mathrm{Cl}, 0.997-0.999)$ \\
\hline
\end{tabular}

gradings. Also, difference and absolute difference between gradings and coefficient of variation of absolute differences were calculated. We calculated the correlation between the preserved EZ area and preserved RPE area using the Pearson correlation coefficient. Correlation between the PAF areas of 2 eyes of the same subject and also correlation between the preserved EZ areas of 2 eyes of the same subject were calculated using Pearson correlation coefficient. Statistical analyses of the data were performed using Statistical Package for Social Science (version 19.0; SPSS, Inc, Armonk, New York, USA) and MedCalc (version 12; MedCalc Software bvba, Mariakerke, Belgium) software.

\section{RESULTS}

A TOTAL OF 148 EYES FROM 75 CASES OF CHM RECEIVED IN DIRC were enrolled in our study.
In 31 eyes (21\%), PAF had extended beyond the FAF captured area. These eyes were excluded from the analysis. In addition, 3 images were not gradable because of low quality. Thus, $114 \mathrm{FAF}$ images were graded.

In $43 \%$ of the eyes, preserved EZ had extended beyond the OCT captured area. After exclusion of these eyes and eyes with low-quality OCT images, a total of 77 OCT images were graded.

PAF and preserved EZ area quantified in independent grading sessions and the results of the comparison between these gradings are shown in Tables 1 and 2 . The level of agreement between gradings is also illustrated in the Bland-Altman plots (Figure 4). The Bland-Altman plots that compare gradings from the 2 independent graders demonstrate that the mean of the difference between measured preserved areas by these 2 graders was $0.03 \mathrm{~mm}^{2}$ and $0.11 \mathrm{~mm}^{2}$ for PAF and preserved EZ areas, respectively, and this difference was less than $0.58 \mathrm{~mm}^{2}$ for PAF and $0.74 \mathrm{~mm}^{2}$ for preserved EZ areas in $95 \%$ of the cases. In addition, the Bland-Altman plots that 

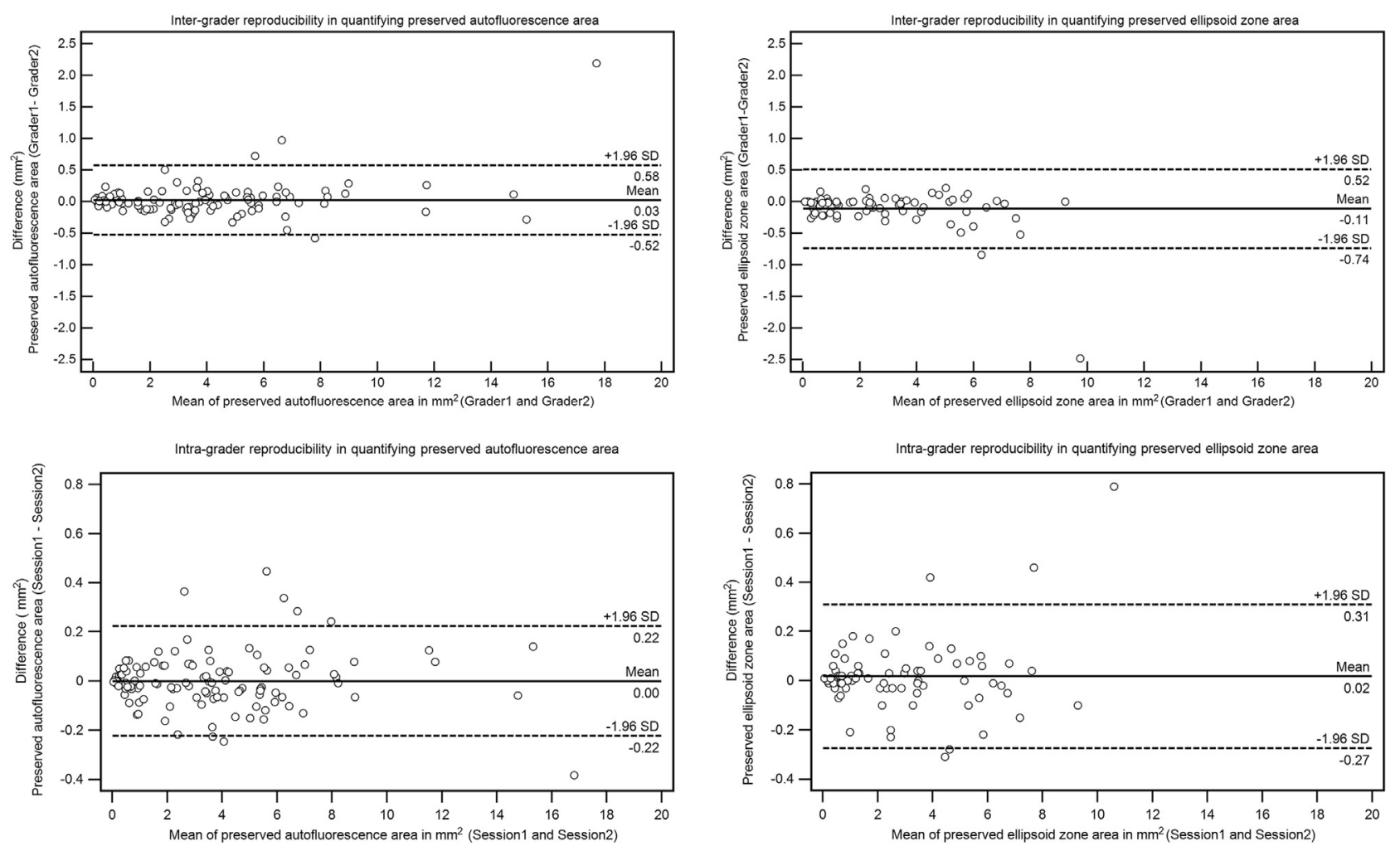

FIGURE 4. (Top) Bland-Altman plots illustrating the level of agreement between 2 independent graders in grading preserved autofluorescence area on fundus autofluorescence (Top left) and preserved ellipsoid zone area on optical coherence tomography (Top right) images of choroideremia patients. (Bottom) In addition, the level of agreement between the 2 different sessions of grading performed by the more senior grader in grading preserved autofluorescence area on fundus autofluorescence images (Bottom left) and preserved ellipsoid zone area on optical coherence tomography images (Bottom right) are illustrated.

compare grading from the 2 different sessions of grading performed by the same grader demonstrate that the mean of the difference between measured preserved areas in these 2 sessions was $0.0 \mathrm{~mm}^{2}$ and $0.02 \mathrm{~mm}^{2}$ for PAF and preserved EZ areas, respectively, and this difference was less than $0.22 \mathrm{~mm}^{2}$ for PAF and $0.31 \mathrm{~mm}^{2}$ for preserved EZ areas in $95 \%$ of the cases.

Post hoc evaluation of eyes with a higher percentage difference between the 2 independent graders showed that preserved areas are much smaller in these cases. We noticed that when the percentage difference was higher than 10 , the PAF area was significantly smaller $\left(1.55 \pm 1.83 \mathrm{~mm}^{2}\right.$ vs $4.00 \pm 3.06 \mathrm{~mm}^{2}, P<.001$ ).

In those eyes in which both preserved EZ and PAF did not extend beyond the captured image area, preserved EZ area was significantly larger than PAF area $(2.66 \pm$ $2.20 \mathrm{~mm}^{2}$ vs $2.51 \pm 2.16 \mathrm{~mm}^{2}$, paired $t$ test, $\left.P<.001\right)$.

In addition, when both preserved EZ and PAF did not extend beyond the captured image area, they were highly correlated (Pearson correlation coefficient $=0.99$, $P<.001$ ). Figure 5 illustrates this correlation.

Figure 6 shows that the PAF areas in 2 eyes of the same subject were highly correlated. A similar high correlation in preserved EZ areas of 2 eyes of the same subject was seen (Pearson correlation coefficient $=0.85, P<.001$, and Pearson correlation coefficient $=0.89, P<.001$, respectively).

\section{DISCUSSION}

IN THIS STUDY, WE IDENTIFIED 2 REPRODUCIBLE METHODS for quantifying anatomic outcome measures for choroideremia clinical trials: segmentation of preserved RPE area and preserved EZ area using FAF and OCT images, respectively. In fact, both the intergrader and intragrader reproducibility in segmenting these preserved structures were excellent. Furthermore, we observed that preserved RPE and EZ areas were highly correlated $(\mathrm{R}=0.99)$, but preserved EZ area was significantly larger than the preserved RPE area in choroideremia patients. The latter finding either implies that in CHM, the RPE degenerates earlier than the photoreceptor layer, leading to the hypothesis that CHM is predominantly a disease of the RPE and secondarily of the photoreceptor layer, or it implies faster degeneration of RPE in the course of the disease regardless of whether the degeneration starts from the outer retina. 


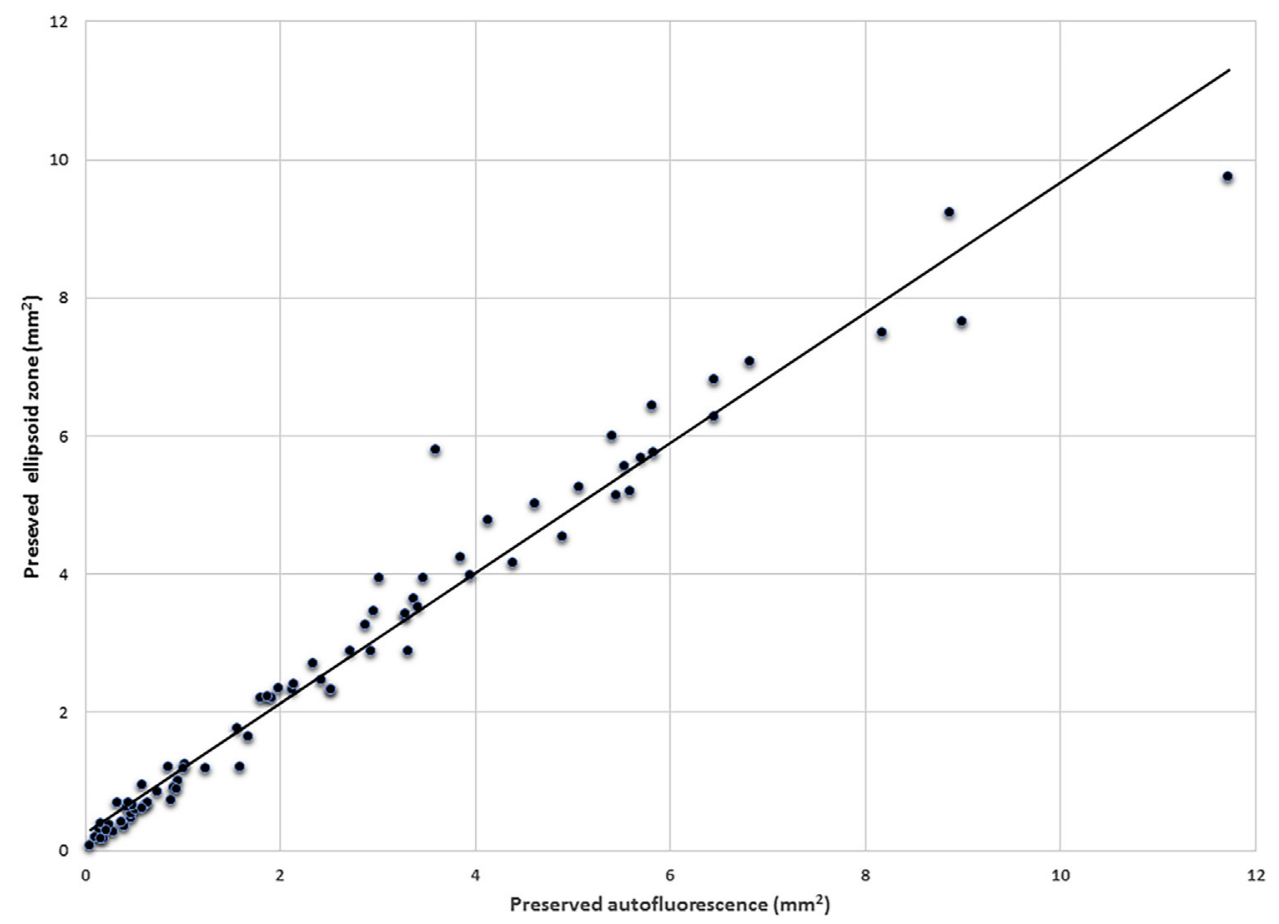

FIGURE 5. Correlation between preserved autofluorescence and preserved ellipsoid zone areas in patients with choroideremia. The means of grader 1 and grader 2 measurements were picked to make the comparison.
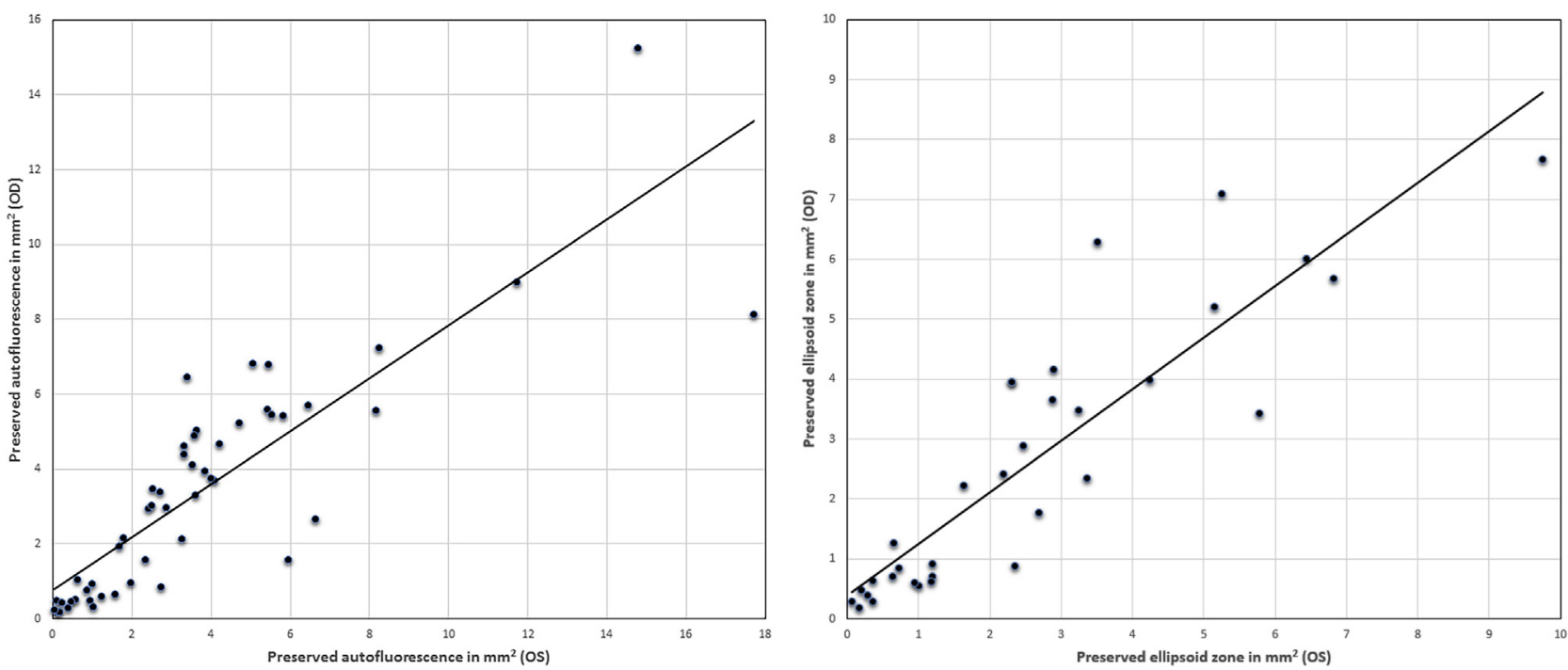

FIGURE 6. Correlation between preserved autofluorescence areas of 2 eyes of the same subject (Left) and between preserved ellipsoid zone areas of 2 eyes of the same subject (Right) in choroideremia.

The results of the first retinal gene therapy for choroideremia patients have recently been published. ${ }^{6}$ The main outcome measure of that study was visual acuity. The current study identifies anatomical outcome measures that can be reproducibly graded if these measures are used to supplement visual acuity data or can be used as a main outcome measure (in lieu of visual acuity) in future and higher-phase clinical trials.

During post hoc review of cases with a higher percentage difference between the 2 independent graders in FAF grading, we noticed that in some of these outlier cases, the PAF brightness was not homogenous; in other words, 

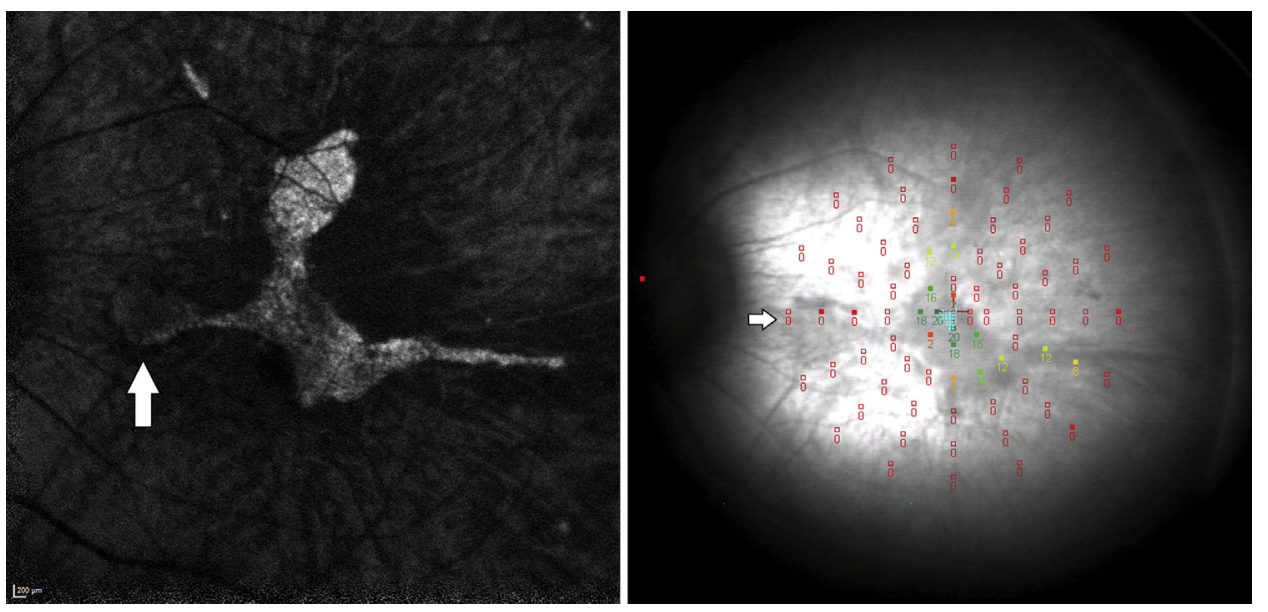

FIGURE 7. An eye with a high percentage difference between the 2 independent graders in fundus autofluorescence grading. (Left) In this fundus autofluorescence image, preserved autofluorescence brightness was not homogenous and the nasal part of preserved autofluorescence (white arrow) was much darker in comparison with the other parts of that. (Right) On microperimetry printout, the corresponding area of this darker part (white arrow) showed zero sensitivity.

some areas at the edge of PAF was much darker in comparison with the other parts of PAF (Figure 7). The corresponding areas of these darker parts of PAF showed zero sensitivity on microperimetry, demonstrating that these areas consist of nonfunctional tissue that might ${ }^{9}$ or might not be viable. Although these fading areas show some preserved autofluorescence, in order to be more reproducible in future studies they likely should not be considered as a preserved area.

Another potential cause of the high percentage difference between the 2 independent graders in FAF grading was the particular pattern of tissue loss in CHM. In most of the cases, the shape of the preserved area was very irregular, with hair-like projections that made it very difficult to mark the exact boundaries of PAF (Figure 1). Particularly in longitudinal studies, it is important to meticulously and consistently segment these hair-like projections.

We noted that in $21 \%$ of eyes the PAF extended beyond the captured area of the FAF image and in $43 \%$ of eyes the preserved EZ extended beyond the captured area of the OCT image. Because overtime shrinkage of the PAF and preserved EZ cannot be completely quantified in these cases, they would not be suitable for longitudinal studies and clinical trials. One future solution could be to capture the FAF image with a 55-degree angle, but this may lead to dispersion of image details that may decrease the grading accuracy. In the future, technologies to improve the transverse resolution of OCT images will help us to expand the range of patients eligible for clinical trials in CHM. Another solution is to acquire multiple steered OCT volumes, which can then be montaged to cover much larger areas of retina. ${ }^{17}$ Swept-source OCT, which rapidly scans larger regions, may also address this issue.
One of the alternative methods for segmenting preserved EZ is taking advantage of en face images. ${ }^{14}$ This method depends on having a reliable segmentation of the boundary between the RPE and Bruch's membrane, because this is the reference structure from which the offsets for the en face slab are selected. However, in $100 \%$ of CHM patients enrolled in this study, the above-mentioned segmentation line failed to be in proper position because the segmentation algorithm graded the high reflectance boundary between choroid and sclera as the RPE band. Furthermore, even after generating an appropriate en face image it was difficult to differentiate pigment hyperplasia from preservation of the EZ on en face images.

High correlation between PAF areas in 2 eyes of the same CHM patient, and between the preserved EZ areas in 2 eyes of the same subject that were observed in this study, would also be important, as in future clinical trials the fellow eyes of the participating subjects could be used as the potential control group.

Our study has some limitations. First, we did not perform a longitudinal study to quantify the progression rate of the disease to compare this rate with grading noise. As shown in previous studies, the rate of shrinkage of PAF is highly correlated with the stage of the disease, with slower progression in more advanced cases (smaller preserved RPE). ${ }^{12}$ Thus, it is possible that in very advanced stages of the disease the rate of the progression of diseases would not be higher than the grading noise. Future clinical trials of CHM could consider enrolling less advanced cases, as this could reduce grading noise and also could serve to enrich the cohort being evaluated (faster progression) to reduce the required sample size. A second limitation is that we did not quantify the actual choroidal circulation, 
as we did not have access to indocyanine green angiography images.

On the other hand, this study has some strengths, including the use of trained, certified reading center graders and demonstration of a high level of reproducibility, which further facilitates its ultimate translation into clinical trials. Another strength of this study is that the cohort studied was large for a relatively uncommon orphan disease. Additionally, extensive imaging was performed and was done by trained and certified personnel at the clinical sites.
In summary, quantifying the preserved RPE area and EZ area in eyes with choroideremia on FAF and OCT images is a reproducible metric that can be studied and tracked longitudinally in CHM. These metrics may be useful for monitoring anatomic changes and assessing the efficacy of new therapeutic approaches for CHM patients. Lastly, these imaging techniques could also be used to follow patients, longitudinally, in the clinical setting, to assist clinicians and patients with monitoring disease progression.

FUNDING/SUPPORT: NO FUNDING OR GRANT SUPPORT. FINANCIAL DISCLOSURES: ANIZ GIRACH IS AN EMPLOYEE OF NIGHTstaRx. Michael S. Ip is a consultant for Thrombogenics, Boehringer Ingelheim, Omeros, Genentech, and Quark. Frank G. Holz is a member of the scientific advisory board for Heidelberg Engineering. He also receives research support from Carl Zeiss Meditec, Pixium, and Optos. He also served as a consultant for Allergan, Bayer, Boerhinger-Ingelheim, Genentech/Roche, GSK, and Novartis. David G. Birch is a consultant for NightstaRx, AGTC, Shire Pharmaceuticals, and Ionis Pharmaceuticals. He is a contractor for Genentech. Robert E. MacLaren is coinventor on a UK patent application filed on February 22, 2011, and owned by the University of Oxford. SriniVas R. Sadda is a co-inventor of Doheny intellectual property related to optical coherence tomography that has been licensed by Topcon Medical Systems, and is a member of the scientific advisory board for Heidelberg Engineering. He also receives research support from Carl Zeiss Meditec, Optos, and Optovue, Inc, and served as a consultant for Genentech, Inc, Regeneron, and Allergan, Inc. The following authors have no financial disclosures: Amir H. Hariri, Swetha B. Velaga, Phuc V. Le, Byron L. Lam, M. Dominik Fischer, Eeva-Marja Sankila, Mark E. Pennesi, Carel B. Hoyng, Ian M. MacDonald, Graeme C. Black, Stephen H. Tsang, Neil M. Bressler, Michael Larsen, Michael B. Gorin, and Andrew R. Webster. All authors attest that they meet the current ICMJE criteria for authorship.

\section{REFERENCES}

1. Van Den Hurk JA, Schwartz M, Van Bokhoven H, et al. Molecular basis of choroideremia (CHM): mutations involving the Rab escort protein-1 (REP-1) gene. Hum Mutat 1997;9(2):110-117.

2. Sankila EM, Tolvanen R, van den Hurk JA, Cremers FP, de la Chapelle A. Aberrant splicing of the CHM gene is a significant cause of choroideremia. Nat Genet 1992;1(2):109-113.

3. Seabra MC, Brown MS, Goldstein JL. Retinal degeneration in choroideremia: deficiency of Rab geranylgeranyl transferase. Science 1993;259(12):377-381.

4. Cremers FP, van de Pol DJ, van Kerkhoff LP, Wieringa B, Ropers HH. Cloning of a gene that is rearranged in patients with choroideraemia. Nature 1990;347:674-677.

5. Jacobson SG, Cideciyan AV, Sumaroka A, et al. Remodeling of the human retina in choroideremia: Rab escort protein 1 (REP-1) mutations. Invest Ophthalmol Vis Sci 2006;47(9): 4113-4120.

6. MacLaren RE, Groppe M, Barnard AR, et al. Retinal gene therapy in patients with choroideremia: initial findings from a phase 1/2 clinical trial. Lancet 2014;383(9923):1129-1137.

7. Chan SC, Bubela T, Dimopoulos IS, Freund PR, Varkouhi AK, MacDonald IM. Choroideremia research: report and perspectives on the second international scientific symposium for choroideremia. Ophthalmic Genet 2016;37(3):267-275.

8. Morgan JI, Han G, Klinman E, et al. High-resolution adaptive optics retinal imaging of cellular structure in choroideremia. Invest Ophthalmol Vis Sci 2014;55(10):6381-6397.

9. Dimopoulos IS, Chan S, Maclaren RE, Macdonald IM. Pathogenic mechanisms and the prospect of gene therapy for choroideremia. Expert Opin Orphan Drugs 2015;3(7): 787-798.

10. Edwards TL, Jolly J, Groppe M, et al. Visual acuity after retinal gene therapy for choroideremia. N Engl J Med 2016; 374(20):1996-1998.

11. Seitz IP, Zhour A, Kohl S, et al. Multimodal assessment of choroideremia patients defines pre-treatment characteristics. Graefes Arch Clin Exp Ophthalmol 2015;253(12): 2143-2150.

12. Nabholz N, Lorenzini M-C, Bocquet B, et al. Clinical evaluation and cone alterations in choroideremia. Ophthalmology 2016;123(8):1830-1832.

13. Nittala MG, Hariri A, Wong WT, Chew EY, Ferris FL, Sadda SR. Image scaling difference between a confocal scanning laser ophthalmoscope and a flash fundus camera. Ophthalmic Surg Lasers Imaging Retina 2015;46(8):872-879.

14. Hariri AH, Zhang HY, Ho A, et al. Quantification of ellipsoid zone changes in retinitis pigmentosa using en face spectral domain-optical coherence tomography. JAMA Ophthalmol 2016;134(6):628-635.

15. Joeres S, Tsong JW, Updike PG, et al. Reproducibility of quantitative optical coherence tomography subanalysis in neovascular age-related macular degeneration. Invest Ophthalmol Vis Sci 2007;48:4300-4307.

16. Sadda SR, Joeres S, Wu Z, et al. Error correction and quantitative subanalysis of optical coherence tomography data using computer-assisted grading. Invest Ophthalmol Vis Sci 2007;48: 839-848.

17. Mori K, Kanno J, Gehlbach PL. Retinochoroidal morphology described by wide-field montage imaging of spectral domain optical coherence tomography. Retina 2016;36(2):375-384. 